\title{
Myocardial injury in non-cardiac surgery: complexities and challenges
}

Navin Kuthiah ${ }^{1}$, MRCP, MSc, Chaozer $\underline{E}^{1}$, MRCP, FAMS

ABSTRACT The term MINS (myocardial injury after non-cardiac surgery) was coined to broadly describe perioperative troponin elevation that is deemed to be due to a cardiac cause. However, this term is commonly used in literature to represent cases that do not fulfil the criteria for the diagnosis of myocardial infarction. Asymptomatic troponin elevation that does not fulfil the criteria for acute coronary syndrome in the perioperative setting has been shown to be associated with increased mortality. The discovery of MINS presents new opportunities to improve outcomes for surgical patients. Unfortunately, awareness of MINS among practitioners remains low and implementation of perioperative troponin monitoring is poor. Given its significance, the detection, management and prevention of MINS should not be overlooked.

Keywords: MINS, myocardial injury, perioperative, troponin

\section{INTRODUCTION}

MINS, referring to myocardial injury after non-cardiac surgery, is a relatively underappreciated new concept that describes myocardial strain in the perioperative period. MINS has gained prominence since the VISION trial highlighted the significant association between MINS and 30-day mortality. ${ }^{(1)}$ This association was described much earlier by Landesberg et al, who showed in 2003 that even low levels of troponin T elevation in the first three days following vascular surgery were correlated with increased five-year mortality. ${ }^{(2)}$ The magnitude of the issue is great. Of the 200 million patients undergoing non-cardiac surgery per year around the world, eight million will suffer from MINS and one million adults are estimated to die within 30 days of surgery, ${ }^{(3)}$ with cardiovascular events contributing to a significant number of deaths. ${ }^{(4)}$ In the VISION trial, the fourth-generation troponin T assay was monitored six, 12, 24, 48 and 72 hours after surgery. ${ }^{(1)}$ While it is known that acute coronary syndrome leads to increased mortality, the trial proved that even troponin levels in the upper range of normal, below the prevailing threshold of $0.04 \mathrm{ng} / \mathrm{mL}$, were associated with increased mortality ${ }^{(1)}$ Since the VISION trial, there have been numerous other studies that substantiated this finding, emphasising the role of routine troponin measurements in surgical patients to enable further perioperative risk stratification and optimisation. ${ }^{(5-7)} \mathrm{A}$ large proportion of patients with MINS are asymptomatic. ${ }^{(5,6)}$

Based on the estimated incremental cost per health gain, utilisation of perioperative troponin monitoring seems to be an attractive proposition, especially in high-risk patients. ${ }^{(8)}$ There are no studies to show that perioperative troponin monitoring is counterproductive. Nonetheless, some authors have argued that it causes more harm, as mild asymptomatic elevations of troponin may be due to non-cardiac factors. For instance, Beckman opined that the lack of treatment strategy and subjecting the patient to treatment of Type 1 myocardial infarction are detrimental. ${ }^{(9)}$

\section{CHALLENGES}

Despite emerging evidence, MINS is widely unrecognised, as there is currently no global consensus on the definition and diagnostic criteria of MINS. Many studies use varying terminology such as POMI (perioperative/postoperative myocardial infarction) $)^{(7)}$ and PMI (perioperative myocardial injury) ${ }^{(5)}$ to describe the phenomenon. Additionally, cut-offs for troponin levels vary in studies depending on the subtype of troponin and the assay used. This heterogeneity makes it challenging to develop a universal definition and guideline.

The next question that arises is: do all patients with perioperative troponin elevation have MINS? Diagnosing MINS can be precarious in patients with elevated baseline troponin levels. Patients with chronic renal failure have been acknowledged to have asymptomatic persistently elevated troponin levels, which are largely assumed to be insignificant. However, studies have shown that these patients have increased fatal cardiovascular events and all-cause mortality compared to renal patients without raised troponin levels. ${ }^{(10)}$ As there are numerous non-cardiac causes of troponin elevation, such as pulmonary embolism, sepsis and pneumonia, there is no clear agreement on when to label a patient with raised perioperative troponin levels as having MINS. Landesberg and Jaffe opined that raised troponin levels are associated with increased mortality; lower levels are associated with non-cardiac complications, whereas higher levels correlate better with cardiac events. ${ }^{(11)}$ More recently, Puelacher et al demonstrated that patients with elevated baseline troponin levels and those with non-cardiac causes for troponin elevation were at higher risk of both cardiovascular and noncardiovascular deaths at 30 days and one year. ${ }^{(5)}$ These findings led to a paradigm shift in the perception of elevated baseline troponin levels and (previously assumed to be insignificant) non-cardiac causes of troponin elevation.

Of the three major perioperative cardiac risk assessment guidelines, the 2014 European Society of Cardiology/European

${ }^{1}$ Department of Internal Medicine, Woodlands Health Campus, Singapore

Correspondence: Dr Navin Kuthiah, Consultant, Department of Internal Medicine, Woodlands Health Campus, 2 Yishun Avenue 2, Singapore 768024 . navinkuthiah@hotmail.com 
Society of Anaesthesiology (ESC/ESA), ${ }^{(12)} 2014$ American College of Cardiology/American Heart Association (ACC/AHA) ${ }^{(13)}$ and 2016 Canadian Cardiovascular Society (CCS) guidelines, ${ }^{(14)}$ only the CCS guideline has integrated daily postoperative troponin measurements for 48-72 hours in patients who are deemed high risk based on revised cardiac risk index scores and brain natriuretic peptide (BNP)/pro-BNP levels ${ }^{(14)}$ (Class I recommendation, Level B evidence). MINS is defined as a peak fourth-generation troponin $\mathrm{T}$ level $\geq 0.03 \mathrm{ng} / \mathrm{mL}^{.{ }^{(4)}}$ The ACC/AHA guidelines state that the benefit of using troponin screening is uncertain for high-risk groups without signs and symptoms of myocardial ischaemia given the lack of an established management strategy. ${ }^{(13)}$

The shortcoming of the CCS guideline is that it does not recommend any supplementary modalities to further risk-stratify or minimise the risk of MINS preoperatively. Even high-risk patients are not subjected to any interventions to reduce the risk of developing MINS. In view of the serious ramification of MINS, high-risk patient groups would benefit from further evaluation, including echocardiography and stress testing. These modalities have been proven to further risk-stratify and identify patients who will benefit from optimisation of medications and preoperative revascularisation, as advocated in the 2014 ESC/ESA ${ }^{(12)}$ and ACC/AHA guidelines ${ }^{(13)}$ (Class Ila recommendation, Level C evidence). However, the decision to revascularise a patient with stable cardiovascular disease should be made judiciously after weighing the benefits against the risks, because there may be no improvement in long-term survival and short-term benefit, as shown in a study by McFalls et al on patients undergoing vascular surgery. ${ }^{(15)}$ Nevertheless, this study may have been influenced by proficiency bias, as the screening and recommendation to revascularise was made by cardiologists at each study site based on their interpretation of the cardiac risk.

\section{MANAGEMENT}

In the management of MINS, the CCS guideline recommends the use of aspirin and statin in patients who do not fulfil the criteria for acute coronary syndrome, as this has been shown to improve outcomes (Class I recommendation, Level B evidence).. ${ }^{(14)}$ Beta blockers and angiotensin blockade are not mentioned in the guidelines. Theoretically, beta blockers may have a role in management to address the supply and demand mismatch that results in MINS. However, the benefits of using beta blockers ${ }^{(16)}$ and angiotensin blockade agents must be weighed against the risk of hypotension, which may accentuate the myocardial injury. Further studies are needed to provide more robust evidence on the use of these drugs. Patients who fulfil the criteria of acute coronary syndrome should be treated as per current clinical practice guidelines.

The MANAGE trial published in 2018 examined the use of dabigatran for the treatment of MINS. It found that subjects in the intervention arm had lower major vascular event rates with similar bleeding complications as compared with the placebo arm. ${ }^{(17)}$ However, this study was flawed: the trial was terminated early, the primary outcome was modified midway through the trial, and drug discontinuation rates were high. Despite its shortcomings, the study opens avenues for further research in this area.

\section{PREVENTION}

A point that remains relatively unaddressed is the prevention of MINS. The pathophysiology of MINS is an area of contention. The proposed mechanisms of myocardial injury in the perioperative period are fissuring or rupture of atherosclerotic plaque, a hypercoagulable state, myocardial oxygen demandsupply mismatch due to hypotension, tachycardia, elevated catecholamine levels and dysrhythmias. ${ }^{(18)}$ As such, in the prevention of MINS, a balance must be attained between providing adequate antithrombotic cover without increasing the risk of bleeding and minimising the adverse effects of the increased sympathetic drive while avoiding hypotension.

The POISE study showed that metoprolol use led to increased mortality rates and incidence of stroke despite reducing the risk of perioperative myocardial infarction. ${ }^{(16)}$ This study is contentious because blanket high-dose metoprolol was served very soon before surgery, resulting in hypotension. Due to a lack of studies proving the benefit of beta blockers in the perioperative period after the discredited DECREASE trials, initiation of antihypertensive agents in the immediate preoperative period is discouraged. ${ }^{(19)}$ Chronic antihypertensive medications should be optimised.

Aspirin addresses the postoperative hypercoagulable state, which can result in ischaemic events, but the role of aspirin in the prevention of MINS remains controversial. Results from the POISE 2 trial showed that aspirin did not result in an improvement in the rate of cardiovascular events and mortality at 30 days, instead causing a significant increase in bleeding compared to a placebo. ${ }^{(20)}$ The authors postulated that the increase in cardiovascular events may be related to increased bleeding, causing a supply-demand mismatch.

However, the POISE 2 trial has some shortcomings that potentially limit its applicability. For one, the duration that aspirin was withheld prior to surgery was not standardised. Based on the inclusion criteria, aspirin would have been indicated for secondary prevention in most of the patients in the trial. Withholding aspirin in these patients may have put them at increased risk of cardiac events and stroke, as withdrawal of chronic aspirin treatment results in a rebound increase of thromboxane A2 and decreases fibrinolysis. ${ }^{(21)} \mathrm{A}$ large meta-analysis demonstrated that discontinuing aspirin perioperatively in patients with or at risk of ischaemic heart disease was associated with a threefold higher risk of major adverse cardiac events. ${ }^{(22)}$ Moreover, some patients in both the aspirin and placebo arms of the POISE 2 trial were on P2Y inhibitors, and more than half of the patients in both groups were given prophylactic anticoagulation. This may have confounded the results of the study, notably in relation to increased bleeding events. There was significantly increased bleeding in the aspirin initiation group as compared to the group of patients who had uninterrupted aspirin. This would have skewed the results to indicate that patients in the aspirin group had a greater risk of bleeding compared to those in the placebo group.

An observational study by George et al showed that preoperative aspirin and statins reduce the risk of developing MINS and mortality rate. ${ }^{(6)}$ All the three major guidelines recommend continuing statin therapy in the perioperative period 
(Class I recommendation, Level C evidence). ${ }^{(12-14)}$ The ESC/ESA ${ }^{(12)}$ and $\mathrm{ACC} / \mathrm{AHA}^{(13)}$ guidelines also recommend initiating statins in patients undergoing vascular surgery (Class Ila, Level B evidence). An observational study of the VISION data by Berwanger et al showed that patients who were taking statin preoperatively had significantly lower rates of MINS and all-cause mortality. ${ }^{(23)}$ Statins should be started at least two weeks prior to surgery to benefit from their plaque stabilising and anti-inflammatory effects. ${ }^{(14)}$

\section{CONCLUSION}

Recent emerging evidence indicates that a rise in perioperative troponin levels is analogous with increased mortality, which makes perioperative troponin a promising modality for perioperative risk assessment. Risk assessment and management of MINS should form an integral part of routine perioperative management. Nevertheless, a universal definition of MINS needs to be agreed upon and the terminology used standardised to prevent confusion among medical practitioners. Further studies are needed to increase awareness of MINS among medical practitioners, to guide appropriate prevention and management of MINS.

Most researchers are in agreement that high-risk patient groups can benefit from perioperative troponin monitoring. The presence of troponin elevation should trigger thorough assessment, risk optimisation and perioperative monitoring. In low-risk groups, elevated troponin levels may merely indicate myocardial strain due to the surgery or severity of the underlying non-cardiac illness. It is still vital to note these cases, as non-cardiac causes of troponin elevation are related to increased mortality. ${ }^{(5)}$ Thus, troponin may be used as a prognostic tool for patients who are critically ill, with the understanding that elevation of troponin is a prognostic indicator of severity of illness and does not directly correlate to ischaemic heart disease. Given this ambiguity, the attending physician needs to establish if the raised troponin level can be attributed to underlying cardiac disease, as it would influence management. This should be done on a case-by-case basis based on the patient profile.

Although the management of patients with MINS from a cardiac cause differs from that of the group without a cardiac cause, both groups of patients will benefit from closer postoperative monitoring. For high-risk patients, the use of aspirin for prevention of MINS in the preoperative period can be cautiously considered. The decision to start aspirin should be made by a multidisciplinary team with the involvement of the patient, after weighing the surgical risk of bleeding against the benefits of preventing MINS. Concurrent use of aspirin with other antiplatelets, nonsteroidal anti-inflammatory drugs and prophylactic anticoagulation must be avoided to minimise bleeding risk. Statins should be given to high-risk patient groups.

\section{REFERENCES}

1. Botto F, Alonso-Coello P, Chan MT, et al; Vascular events In noncardiac Surgery patlents cOhort evaluatioN (VISION) Writing Group, on behalf of The Vascular events In noncardiac Surgery patlents cOhort evaluatioN (VISION)
Investigators; Appendix 1. The Vascular events In noncardiac Surgery patlents cOhort evaluatioN (VISION) Study Investigators Writing Group; Appendix 2. The Vascular events In noncardiac Surgery patlents cOhort evaluatioN Operations Committee; Vascular events In noncardiac Surgery patlents cOhort evaluatioN VISION Study Investigators. Myocardial injury after noncardiac surgery: a large, international, prospective cohort study establishing diagnostic criteria, characteristics, predictors, and 30-day outcomes. Anaesthesiology 2014; 120:564-78.

2. Landesberg G, Shatz V, Akopnik I, et al. Association of cardiac troponin, CK-MB and postoperative myocardial ischemia with long-term survival after major vascular surgery. J Am Coll Cardiol 2003; 42:1547-54

3. Vascular Events In Noncardiac Surgery Patients Cohort Evaluation (VISION) Study Investigators, Devereaux PJ, Chan MT, et al. Association between postoperative troponin levels and 30-day mortality among patients undergoing noncardiac surgery. JAMA 2012; 307:2295-304.

4. Devereaux PJ, Xavier D, Pogue J, et al; POISE (PeriOperative ISchemic Evaluation) Investigators. Characteristics and short-term prognosis of perioperative myocardial infarction in patients undergoing noncardiac surgery: a cohort study. Ann Intern Med 2011; 154:523-8.

5. Puelacher C, Lurati Buse G, Seeberger D, et al; BASEL-PMI Investigators. Perioperative myocardial injury after noncardiac surgery: incidence, mortality, and characterization. Circulation 2018; 137:1221-32.

6. George R, Menon VP, Edathadathil F, et al. Myocardial injury after noncardiac surgery-incidence and predictors from a prospective observational cohort study at an Indian tertiary care centre. Medicine (Baltimore) 2018; 97:e0402.

7. van Waes JA, Nathoe HM, de Graaff JC, et al; Cardiac Health After Surgery (CHASE) Investigators. Myocardial injury after noncardiac surgery and its association with short-term mortality. Circulation 2013; 127:2264-71.

8. Buse GL, Manns B, Lamy A, et al. Troponin T monitoring to detect myocardial injury after noncardiac surgery: a cost-consequence analysis. Can J Surg 2018; 61:185-94.

9. Beckman JA. Postoperative troponin screening: a cardiac Cassandra? Circulation 2013; 127:2253-6.

10. Freda BJ, Tang WH, Van Lente F, Peacock WF, Francis GS. Cardiac troponins in renal insufficiency: review and clinical implications. J Am Coll Cardiol 2002; 40:2065-71.

11. Landesberg G, Jaffe AS. 'Paradox' of troponin elevations after non-cardiac surgery. Br J Anaesth 2015; 114:863-5.

12. Kristensen SD, Knuuti J, Saraste A, et al; Authors/Task Force Members. 2014 ESC/ESA Guidelines on non-cardiac surgery: cardiovascular assessment and management: The Joint Task Force on non-cardiac surgery: cardiovascular assessment and management of the European Society of Cardiology (ESC) and the European Society of Anaesthesiology (ESA). Eur Heart J 2014; 35:2383-431.

13. Fleisher LA, Fleischmann KE, Auerbach AD, et al. 2014 ACC/AHA guideline on perioperative cardiovascular evaluation and management of patients undergoing noncardiac surgery: executive summary: a report of the American College of Cardiology/American Heart Association Task Force on Practice Guidelines. Circulation 2014; 130:2215-45.

14. Duceppe E, Parlow J, MacDonald P, et al. Canadian Cardiovascular Society guidelines on perioperative cardiac risk assessment and management for patients who undergo noncardiac surgery. Can J Cardiol 2017; 33:17-32.

15. McFalls EO, Ward HB, Moritz TE, et al. Coronary-artery revascularization before elective major vascular surgery. N Engl J Med 2004; 351:2795-804

16. POISE Study Group, Devereaux PJ, Yang H, et al. Effects of extended-release metoprolol succinate in patients undergoing non-cardiac surgery (POISE trial): a randomised controlled trial. Lancet 2008; 371:1839-47.

17. Devereaux PJ, Duceppe E, Guyatt G, et al; MANAGE Investigators. Dabigatran in patients with myocardial injury after non-cardiac surgery (MANAGE): an international, randomised, placebo-controlled trial. Lancet 2018; 391:2325-34.

18. Grobben RB, van Klei WA, Grobbee DE, Nathoe HM. The aetiology of myocardial injury after non-cardiac surgery. Neth Heart J 2013; 21:380-8.

19. Cole GD, Francis DP. Perioperative $\beta$ blockade: guidelines do not reflect the problems with the evidence from the DECREASE trials. BMJ 2014; 349:g5210.

20. Devereaux PJ, Mrkobrada M, Sessler DI, et al; POISE-2 Investigators. Aspirin in patients undergoing noncardiac surgery. N Engl J Med 2014; 370:1494-503.

21. Oscarsson A, Gupta A, Fredrikson M, et al. To continue or discontinue aspirin in the perioperative period: a randomized, controlled clinical trial. $\mathrm{Br}$ J Anaesth 2010; 104:305-12.

22. Biondi-Zoccai GG, Lotrionte $M$, Agostoni $P$, et al. A systematic review and meta-analysis on the hazards of discontinuing or not adhering to aspirin among 50,279 patients at risk for coronary artery disease. Eur Heart J 2006; 27:2667-74.

23. Berwanger O, Le Manach Y, Suzumura EA, et al; VISION Investigators. Association between pre-operative statin use and major cardiovascular complications among patients undergoing non-cardiac surgery: the VISION study. Eur Heart J 2016; 37:177-85. 